\title{
APLICAÇÃO DE TÉCNICAS COMPUTACIONAIS EM BASES DE DADOS DE TRANSPLANTE: REVISÃO DE ARTIGOS PUBLICADOS NO BIÊNIO 2007-2008
}

\author{
Computer Techniques Applied to Transplant Database: Review of Articles Published \\ in the 2007-2008 Biennium
}

\author{
Anderson Diniz Hummel', Rafael Fabio Maciel'2, Alex Esteves Jaccoud Falcão', Fabio Teixeira', Felipe Mancini', \\ Thiago Martini da Costa', Frederico Molina Cohrs², Fernando Sequeira Sousa', Ivan Torres Pisa ${ }^{3}$
}

\begin{abstract}
RESUMO
Introdução: Há séculos, a Humanidade preocupa-se em substituir órgãos defeituosos por outros sadios, mas somente há poucas décadas os avanços da Medicina tornaram esse sonho realidade, por meio dos transplantes de órgãos. Com o intuito de melhorar o entendimento dos processos envolvidos e até mesmo detectar qual perfil de paciente receptor terá maior chance de sobrevida, diversas técnicas de análise de base de dados de transplantes de órgãos têm sido empregadas, contribuindo de forma direta ou indireta na descoberta de conhecimento ainda não mapeado. Objetivo: Apresentar, a partir da literatura científica especializada, quais técnicas computacionais estão sendo utilizadas para analisar bases de dados de transplante de órgãos. Métodos: Para revisão bibliográfica foi realizada pesquisa em resumos de artigos publicados no biênio 2007-2008, utilizando bases de dados científicas PubMed e ISI. Resultados: Foram selecionados 89 resumos de artigos e, após análise dos resumos considerando os critérios de inclusão e exclusão, foram selecionados cinco artigos. Esses mostravam que redes neurais artificiais e regressão logística obtêm bons resultados quando aplicados em bases de dados de transplante de órgãos para descoberta de conhecimento e, de forma geral, apresentavam resultados animadores em suas respectivas bases de dados de estudo. Poucos estudos realmente foram aplicados na prática clínica. Conclusão: Tendo em vista os resultados encontrados na literatura, a aplicação de técnicas de inteligência artificial tem obtido resultados superiores às normalmente utilizadas para previsão em dados de transplante e existe potencial para desenvolvimento de sistemas de apoio à decisão clínica para transplante de órgãos.
\end{abstract}

Descritores: Informática em Saúde, Inteligência Artificial, Transplante de Órgãos, Revisão.

\footnotetext{
Instituições:

1 Programa de Pós-graduação em Informática em Saúde, Universidade Federal de São Paulo (UNIFESP) - São Paulo / SP

2 Programa de Pós-graduação em Saúde Coletiva, UNIFESP - São Paulo / SP

${ }^{3}$ Departamento de Informática em Saúde, UNIFESP - São Paulo / SP
}

Correspondência:

Anderson Diniz Hummel

Rua Botucatu, 862 - Vila Clementino - São Paulo / SP - CEP: 04023-900 - Brasil

Tel.: (11) 55740110

E-mail: anderson.hummel@unifesp.br

\section{INTRODUÇÃO}

Há séculos, a Humanidade preocupa-se em substituir órgãos defeituosos por outros sadios, mas somente há poucas décadas os avanços da Medicina tornaram esse sonho realidade por meio dos transplantes de órgãos. ${ }^{1,2}$

No entanto, essa área desponta como um cenário complexo no qual o transplante de órgãos está associado, tanto quanto ao aumento da qualidade de vida e sobrevida do paciente, como também à baixa oferta de órgãos, consideráveis taxas de rejeição e de mortalidade na fila de espera, nefrotoxicidade dos imunossupressores, alto custo do procedimento e dificuldades na captação do órgão. ${ }^{3-14}$

Logo, melhorias nos procedimentos de triagem, captação e pré e pós-operatório no transplante de órgãos têm tido cada vez mais importância na prática clínica, principalmente quando se discute a diminuição do insucesso ou melhorias na qualidade de vida do paciente.

Com o intuito de melhorar o entendimento dos processos envolvidos e até mesmo detectar qual perfil de paciente receptor terá maior chance de sobrevida, diversas técnicas de análise de 
base de dados de transplantes de órgãos têm sido empregadas, contribuindo de forma direta ou indireta na descoberta de conhecimento ainda não mapeado..$^{10,15,16}$

Nesse contexto, estão inseridos sistemas de auxílio à decisão clínica (SADCs). Os SADCs, definidos por Berner como "algoritmos computacionais que auxiliam o médico com um ou mais passos que compõem o processo de diagnóstico clínico", colaboram com a descoberta de conhecimento, por vezes superando até mesmo as dificuldades intrínsecas à própria natureza do conhecimento médico. Como exemplo, os SADCs podem ser utilizados como ferramentas que identifiquem a gravidade de uma doença ou o melhor tratamento, ajudando o médico a tomar a melhor decisão para cada caso. ${ }^{17-21}$

$\mathrm{Na}$ literatura existem exemplos de artigos publicados que aplicaram técnicas matemáticas para o desenvolvimento de SADCs; dentre essas técnicas podem-se citar: análise estatística, aplicação de modelos matemáticos e aplicação de técnicas de inteligência artificial. ${ }^{10,16,22,23}$

\section{OBJETIVO}

O objetivo deste artigo é apresentar, a partir da literatura científica especializada, quais técnicas computacionais estão sendo utilizadas para analisar bases de dados de transplante de órgãos.

\section{MÉTODOS}

Foi realizada uma pesquisa bibliográfica a partir das seguintes bases de dados: PubMed (http://www.ncbi.nlm.nih.gov/pubmed) e ISI (http://isiknowledge.com). Na busca por artigos científicos foram utilizadas palavras-chave que relacionam técnicas computacionais a transplantes de órgãos nos campos de título e resumo. Foram selecionados dois grupos de termos: médicos e computacionais. O primeiro grupo incluiu o termo transplante de órgão (transplantation) do vocabulário do Medical Subject Headings (MeSH). Já para o segundo grupo foram selecionados termos relacionados à inteligência artificial (Artificial intelligence, Decision support systems, Artificial Neural Networks, Decision trees, Fuzzy logic, Decision support techniques). As buscas realizadas são apresentadas a seguir:

Para o PubMed: "Transplantation"[Mesh] AND (((("artificial intelligence"[MESH] OR "Decision support systems, clinical"[MESH]) OR "Neural Networks (Computer)"[Mesh]) OR ("Decision Trees/methods"[Mesh] OR "Decision Trees/statistics and numerical data"[Mesh]) ) OR "Fuzzy Logic"[Mesh] ) OR "Decision Support Techniques"[Mesh] ).

Para o ISIWeb: Topic $=($ "Transplantation" AND ( "artificial intelligence" OR "Decision support systems" OR "Artificial Neural Networks" OR "Decision Trees" OR "Fuzzy Logic" OR "Decision Support Techniques" ).

Todos os resumos de artigos retornados pelos buscadores foram lidos e analisados segundo os seguintes critérios de inclusão: ter sido publicado no biênio 2007-2008; informar no resumo explicitamente se a base de dados analisada é de transplante; utilizar técnicas computacionais ou matemáticas para análise de dados e estar em língua inglesa. Os critérios de exclusão considerados foram: excluir artigo que não apresentasse a versão completa para leitura; excluir artigo de revisão da literatura.

\section{RESULTADOS}

Inicialmente foram encontrados 89 artigos nas bases de dados utilizadas nesta pesquisa, tendo sido selecionados para leitura completa cinco artigos, tendo em vista os critérios de inclusão e exclusão. A Tabela 1 ilustra a distribuição por base de dados dos resumos encontrados e dos resumos que foram selecionados para leitura completa.

Tabela 1. Artigos encontrados nas bases de dados ISI e PubMed.

\begin{tabular}{lccc}
\hline & PubMed & ISI & Total \\
\hline Resumos encontrados & 84 & 5 & 89 \\
Resumos selecionados & 3 & 2 & 5 \\
\hline
\end{tabular}

A seguir, são apresentados os métodos e os resultados dos outros cinco artigos.

As técnicas de inteligência artificial utilizadas nos cinco artigos resultantes são: Redes Neurais Artificiais (RNAs) e Análise de Correspondência Múltipla (MCA, de Multiple Correspondece Analysis).

RNAs são sistemas de equações que imitam a topologia dos neurônios no cérebro, sendo necessário treinamento de RNA a partir de um conjunto de dados para que ela possa predizer um determinado comportamento. . $^{16,24}$

A técnica MCA é um método de exploração de dados categóricos utilizada para verificar a correlação de duas ou mais variáveis a partir da análise de um conjunto de dados. ${ }^{26}$

O estudo de Costa aplicou MCA em uma base de dados de 556 transplantes de rins do Hospital Universitário Pedro Ernesto, Rio de Janeiro no período de janeiro de 1986 a dezembro de 1994. Utilizando essa técnica, foram obtidos agrupamentos de pacientes a partir de dados que correspondem ao conhecimento médico atual em relação ao sucesso do transplante, tais como: número de transfusões e HLA idêntico. ${ }^{27}$

O artigo de Cucchetti compara o desempenho do Model End Stage Liver Disease (MELD) em relação a uma RNA para predizer a mortalidade de pacientes na lista de espera por transplante de fígado. Para isso, foi construída e treinada uma RNA em uma base de dados de 251 pacientes com cirrose da Liver Transplant Unit de Bologna, Itália, e que estão na lista de espera para transplante de fígado. A avaliação do modelo foi feita utilizando uma base de dados de 137 pacientes do King's College Hospital de Londres, Inglaterra. Vale ressaltar que esse estudo mostra de maneira consistente que o modelo desenvolvido utilizando RNA foi superior ao MELD para avaliar o risco de mortalidade de pacientes com área sob a curva ROC de 0.95 (IC: 0.86-0.99) e 0,85 (IC: 0.80-0.91) respectivamente e p-valor de $0,02 .^{28}$

No artigo de Akl foi treinada uma RNA para predizer a sobrevivência de cinco anos de doadores renais vivos em 1900 pacientes do Urology and Nephrology Center de Mansoura, Egito. Os resultados do modelo construído foram comparados aos resultados da regressão linear de Cox baseada em nanogramas. Nesse contexto, a RNA possui acurácia de 88\% (CI: 87\%-90\%) e a regressão de Cox de 72\% (CI 67\%-77\%) com intervalo de confiança de $95 \% .^{29}$ 
No estudo de Santori foi construída uma RNA para prever o decaimento do nível sérico de creatina em crianças receptoras de rim. Esse estudo foi realizado com 107 pacientes do Departamento de Transplantes do San Martino University Hospital, Gênova, Itália. Os modelos descritos apresentaram resultados superiores à aplicação das técnicas de regressão logística; os modelos de RNA desenvolvidos possuíam taxa de acerto de $87,14 \%$ (IC: $81 \%$ 90\%) contra 79,05\% (IC: 73,6\%-83.1\%) das técnicas de regressão logística, com intervalo de confiança de $95 \%$. Em contrapartida, os modelos desenvolvidos não foram aplicados em uma base de dados com maior número de pacientes ou de outro centro de transplantes, o que prejudica a avaliação dos resultados deste artigo. ${ }^{30}$

O estudo de Lin compara o uso das técnicas RNA, regressão logística e regressão de Cox para predizer a sobrevivência no transplante de fígado a partir da base de dados da United States Renal Data System, EUA, que coleta dados demográficos de pacientes de transplantes renais. Neste artigo, demonstrou-se que uma técnica é superior à outra, dependendo das interações entre os atributos e da referência temporal (curto ou longo prazo). Como exemplo, a RNA foi superior à regressão logística e ao modelo de Cox na previsão de sobrevida em cinco anos, com taxas de acerto de 77\% (IC: 76\%-78), 75\% (IC: $74-76 \%$ ) e $71 \%$ (IC: $70-72 \%$ ) respectivamente. ${ }^{31}$

\section{DISCUSSÃO}

A aplicação das técnicas de inteligência artificial na área de saúde auxilia a descoberta do conhecimento intrínseco à base de dados e a utilização de ferramentas computacionais que auxiliem na identificação da gravidade da doença ou do melhor tratamento a ser oferecido ao paciente, com a finalidade de auxiliar o médico a tomar a melhor decisão para cada caso. ${ }^{20,21}$

Em bases de dados de transplantes evidencia-se o uso de técnicas de inteligência artificial, principalmente as técnicas de RNAs, destacada nos estudos de Wang e Cucchetti. Ambos os estudos demonstram que uma abordagem baseada em RNA apresenta maior acurácia que o uso da regressão logística em relação ao MELD. Estes resultados corroboram constatações anteriores que detectavam uma acurácia superior das RNAs em comparação às metodologias já existentes, como MELD e Child-Pugh. ${ }^{16,28,32,33}$

Entretanto, Ghoshal ressalta a importância de se realizar estudos aprofundados para que um sistema de apoio à decisão clínica baseado em RNA seja aplicado na prática clínica. Ele ressalta que, embora a aplicação de RNA tenha resultado em uma melhor acurácia que outro protocolo usado, por exemplo, MELD e ChildPugh baseados em regressão logística, em geral, os estudos são focados na efetividade da aplicação da técnica e não nos resultados de sua aplicação na prática clínica. ${ }^{33,34,35}$
Alguns estudos focam a predição de sobrevida do enxerto ou do paciente, como os estudos de Akl e Lin. No estudo de Akl, por exemplo, é demonstrada a superioridade de um modelo de RNA em relação ao modelo de Cox para predizer a sobrevivência do enxerto de rim quando analisadas a acurácia e a sensibilidade de ambos os modelos. ${ }^{31,33}$

Vale ressaltar que técnicas de inteligência artificial extraem mais que o conhecimento já mapeado, pois extraem o conhecimento intrínseco aos dados. O que torna o conhecimento do motor de inferência de um Sistema de Apoio à Decisão Clínica (SADC) extremamente poderoso e útil na prática médica.

Todavia, alguns artigos devem ser analisados com cautela, pois a avaliação dos resultados é dependente das bases de dados utilizadas no treinamento, que podem não refletir a mesma aplicabilidade na prática clínica. Como exemplo, tem-se que a análise estatística de pequenas bases de dados biomédicos pode ser enviesada por eventos de baixa freqüência. Dentre os artigos analisados, alguns deles citaram ter usado pequenas bases de dados. ${ }^{22}$

O estudo de Costa foi o único estudo realizado utilizando bases de dados brasileiras; em contrapartida, ele não objetiva o desenvolvimento de um SADC. Dos 89 artigos encontrados, apenas dois artigos eram referentes à base de dados brasileiras, um número pequeno de artigos tendo em vista o fato de o Brasil ser um dos países com maior número de transplantes no mundo. ${ }^{27}$

\section{CONCLUSÃO}

As técnicas computacionais têm ganhado cada vez mais espaço para análise de dados de transplante. Os artigos publicados recentemente mostram que a aplicação de técnicas de inteligência artificial em base de dados de transplante tem obtido bons resultados em comparação a outras abordagens. Embora poucos estudos tenham sido aplicados na prática clínica, nos trabalhos analisados é observado o potencial para o desenvolvimento e validação de sistemas de apoio à decisão clínica.

Vale ressaltar que esta revisão contribuiu para a escolha estratégica de técnicas utilizadas em alguns trabalhos desenvolvidos na UNIFESP para construção de um sistema de apoio à decisão para transplante de órgãos, usando bases de dados de transplantes brasileiros.

\section{Agradecimentos}

Um agradecimento especial às pessoas envolvidas indiretamente neste projeto, ao Departamento de Informática em Saúde pelo apoio institucional, e à VTEL pelo auxilio no processamento dos dados.

\section{ABSTRACT}

For centuries, the human kind has been worrying about replacing defective organs by healthy ones, but only a few decades ago advancements in organ transplantation has made that dream come true. In order to improve the comprehension process and to detect patients whom will have better survival chances, different techniques to assess the organ transplantation database have been used, thus directly or indirectly contributing to discover the not yet mapped knowledge. Purpose: The purpose of this paper is to present from the specialized literature which computational techniques are being used to assess the organ transplantation databases. Methods: it was conducted a review in the literature using the PubMed and ISI databases in abstracts published in the 2007-2008 biennium. Results: After reviewing al abstracts, we selected 89 abstracts, and upon considering the inclusion and exclusion criteria, we selected 5 articles. These have shown that artificial neural networks 
1048 Anderson Diniz Hummel, Rafael Fabio Maciel, Alex Esteves Jaccoud Falcão, Fabio Teixeira, Felipe Mancini,Thiago Martini da Costa, Frederico Molina Cohrs, Fernando Sequeira Sousa, Ivan Torres Pisa.

and logistic regression obtain good results when applied to organs transplantation databases. These articles have demonstrated encouraging results in their respective study databases. Few studies have actually been applied in the clinical practice. Conclusion: When considering the results, the artificial intelligence application techniques attained better results than the normally used techniques to predict based on the transplantation database, and there is potential to the development of clinical decision support system for organ transplantation.

Keywords: Medical Informatics, Artificial Intelligence, Organ Transplantation; Review.

\section{REFERÊNCIAS}

1 Lazzaretti CT. Dádiva da contemporaneidade: doação de órgãos em transplante intervivos. Epistemo-somática. 2007 Jul;4(1):50-61.

2 Küss R, Bourget P. An Illustrated History Of Organ Transplantation: The Great Adventure Of The Century. France: Laboratoires Sandoz, 1992.

3 Starzl E, Klintmalm GB, Porter KA, Iwatsuki S, Schroter GP. Liver transplantation with use of cyclosporin a and prednisone. N Engl J Med. 1981 Jul 30;305(5):266-9.

4 Perez RV, Matas AJ, Gillingham KJ, Payne WD, Canafax DM, Dunn DL, Gores PF, Sutherland DE, Najarian JS. Lessons learned and future hopes: three thousand renal transplants at the University of Minnesota. Clin Transpl. 1990:217-31.

5 Arent S, Mallat M, Westendorp R, van der Woude F, van Es L. Patient survival after renal transplantation; more than 25 years follow-up. Nephrology Dialysis Transplantation, 1997;12(8):1672-9.

6 Perosa M, Genzini T, Gil AO, Goldestein PJG, Pandullo F, Fornasari G, et al. Transplante de pâncreas isolado (órgão total) com drenagem vesical: relato do primeiro caso do Brasil. Arq Bras Endocrinol Metab. 1999;43(5):393-8.

7 Tacconi MRO, Lee MP, Gomes AEO, Miranda MP, Genzini T, Rodrigues JJG Transplante de pâncreas: relato de caso e revisão da literatura. Rev Medicina. 1997;76(4):235-48.

8 Perosa M, Genzini T. Pancreas-kidney transplantation in Brazil: current difficulties and perspectives. Tranplant Proc. 1999;31(7):3005-6.

9 Moraes EL, Silva LBB, Glezer M, Paixão NCS, Moraes TC. Trauma e a doação de órgãos e tecidos para transplante. JBT J Bras Transpl. 2006;9:561-5

10 Marinho A. A study on organ transplantation waiting lines in Brazil's Unified National Health System. Cadernos de Saúde Pública. 2006 Oct;22(10):2229-39.

11 Kim WR, Therneau TM, Benson JT, Kremers WK, Rosen CB, Gores GJ, Dickson ER. Deaths on the Liver Transplant Waiting List: An Analysis of Competing Risks. Hepatology. 2006;43(2):345-51

12 Freeman Jr RB, Edwards EB. Liver Transplant Waiting Time Does Not Correlate With Waiting List Mortality: Implications for Liver Allocation Policy. Liver Transplantation. 2000 Sep;6(5):543-52.

13 Brasil IRC, Garcia JHP, Viana CFG, Rocha TDS, Vasconcelos JBM, Costa PEG, et al. Fatores associados a mortalidade precoce em lista de espera de transplante hepático em pacientes com pontuação MELD baixa. J Bras Transpl. 2006;9:522-4

14 Fryer J, Pellar S, Ormond D, Koffron A, Abecassis M. Mortality in Candidates Waiting for Combined Liver-Intestine Transplants Exceeds That for Other Candidates Waiting for Liver Transplants Liver Transplantation. 2003 Jul;9(7):748-53.

15 Mcgarry K, A survey of interestingness measures for knowledge discovery. The Knowledge Engineering Review. 2001 Mar;20(1):39-61.

16 Banerjee R, Das A, Ghoshal UC, Sinha M. Predicting mortality in patients with cirrhosis of liver with application of neural network technology. J Gastroenterol Hepatol. 2003;18:1054-60.

17 Berner, ES Clinical Decision Support Systems: Theory and Practice. New York: Springer. 1999

18 Bouchon-Meunier B. Uncertainty Management in Medical Applications. In: Akay, M. (Ed.), Nonlinear Biomedical Signal Processing: Fuzzy Logic, Neural Networks, and New Algorithms. New York: IEEE Press, 2000;1:1-26.

19 Álvaro Rocha. Informática de Saúde - Boas Práticas e Novas Perspectivas. Porto Portugal: Editora da Universidade Fernando Pessoa, 2007.

20 Hunt D, Haynes R, Hanna S, Smith K. Effects of computer-baseddecision support systems on physician performance and patient outcomes. JAMA. 1998;280:1339-46.

21 Blumenthal D. The future of quality measurement and management in a transforming health care system. Journal of the American Medical Association. 1997;278:1622-5

22 Patel S, Cassuto J, Orloff M, Tsoulfas G, Zand M, Kashyap R, Jain A, Bozorgzadeh A, Abt P. Minimizing morbidity of organ donation: analysis of factors for perioperative complications after living-donor nephrectomy in the United States. Transplantation. 2008 Feb;85(4):561-5

23 Murugan R, Venkataraman R, Wahed AS, Elder M, Hergenroeder G, Carter M, et al. HIDonOR Study Investigators. Increased plasma interleukin-6 in donors is associated with lower recipient hospital-free survival after cadaveric organ transplantation. Critical Care Medicine. 2008 Jun;36(6):1810-6.

24 Haykin S. Neural Networks: A Comprehensive Foundation. Upper Saddle River: Prentice Hall. 1999

25 Mohamad HH. Fundamentals of Artificial Neural Networks. Cambridge: MIT Press, 1995.

26 Greenacre MJ, Blassius J. Correspondence analysis in practice. Barcelona: Academic Press, 1993.

27 Costa JCGD, Almeida RMVR, Infantosi AFC, Suassuna JHR. A heuristic index for selecting similar categories in multiple correspondence analysis applied to living donor kidney transplantation. Computer Methods and Programs in biomedicine. 2008 June;90(3):217-29

28 Cucchetti A, Vivarelli M, Heaton ND, Phillips S., Piscaglia F., Bolondi L., La Barba G., Foxton M.R., Rela M., O’Grady J., Pinna A.D. Artificial neural network is superior to MELD in predicting mortality of patients with end-stage liver disease. Gut. 2007;56:253-8.

29 Akl A, Ismail AM, Ghoneim M. Prediction of Graft Survival of Living-Donor Kidney Transplantation: Nomograms or Artificial Neural Networks? Transplantation. 2008;86:1401-6.

30 Santori G, Fontana I, Valente U. Application of an Artificial Neural Network Model to Predict Delayed Decrease of Serum Creatinine in Pediatric Patients After Kidney Transplantation. Transplantation Proceedings. 2007,39,1813-9

31 Lin RS, Horn SD, Hurdle JF, Goldfarb-Rumyantzev AS. Single and multiple timepoint prediction models in kidney transplant outcomes. J Biomed Inform. 2008 Dec;41(6):944-52.

32 Wang CH, Mo LR, Lin RC, Kuo JJ, Chang KK, Wu JJ. Artificial Neural Network Model Is Superior to Logistic Regression Model in Predicting Treatment Outcomes of Interferon-Based Combination Therapy in Patients with Chronic Hepatitis C. Intervirology. 2008;51:14-20.

33 Ghoshal UC, Das A. Models for prediction of mortality from cirrhosis with special reference to artificial neural network: a critical review. Hepatology International. 2008; $2: 31-8$

34 Forman L, Lucey M. Predicting the prognosis of chronic liver disease: an evolution from Child to MELD. Hepatology. 2001;33:473-5

35 Infante-Rivard C, Esnaola S, Villeneuve JP. Clinical and statistical vlidity of conventional prognostic factors in predicting short-term survival among cirrhotics. Hepatology. 1987;7:660-4. 